\title{
Impact of a Standardized Emergency Department Pain Protocol for Children with Sickle Cell Disease
}

\author{
Alexis Booker ${ }^{1}$, Olufunke Y. Martin ${ }^{2}$, Aaron E. Carroll ${ }^{1}$, Seethal A. Jacob² \\ ${ }^{1}$ Indiana University School of Medicine, ${ }^{2}$ Children's National Hospital, Washington D.C.
}

Background/Objective: Sickle cell disease (SCD) is a common heritable red blood cell disorder that transcends the lifespan. Abnormal sickle-shaped erythrocytes interfere with blood flow in small vessels resulting in vaso-occlusion. This vaso-occlusion leads to tissue ischemia and inflammation which causes severe pain and is commonly referred to as a vaso-occlusive crisis (VOC). VOC is the most common complication seen in SCD and accounts for the majority of Emergency Department (ED) visits and hospitalizations. The primary objective of this study was to evaluate if pain management of SCD in pediatric patients at our institution was in accordance with national and institutional pain management guidelines before and after implementation of a standardized pain management algorithm.

Methods: We retrospectively analyzed electronic medical record (EMR) data at Riley Hospital for Children between June 2015-May 2018, as well as from January 2019-December 2019 from for all pediatric patients (0-21 years old) who presented to the Riley ED with a confirmed diagnosis of SCD and uncomplicated VOC. These timeframes represented pre and post implementation of a standardized pain management algorithm, which has been described separately. The data collected included time to first dose of analgesia, type and dose of analgesia, change in pain score, length of stay in the ED, and disposition (discharge or admission). Univariate analyses were used to compare data between encounters.

Results: Prior to implementation, 186 patients were seen for a total of 504 encounters, the majority of whom were male $(59 \%, p<0.001)$ and had $\mathrm{Hb}$ SS disease $(67 \%, p<0.001)$. The mean length of an ER visit was approximately 5 hours ( $+/-113$ minutes). The median pain score at presentation was 9 (0-10 scale), with an average of 23 minutes from check in to first documented pain score. However, $19 \%$ of patients had no initial pain score documented, which occurred more often if the patient's initial analgesic was given orally $(p<0.001)$. The mean time from ED check in to first dose of analgesia (PO, IV, IN) was 76 minutes. The mean time between pain scores and medication administration increased between subsequent doses. $70 \%$ of these encounters resulted in hospitalization, with an average LOS of 4.1 days. Analysis of data following implementation of a standardized pain management algorithm is ongoing.

Conclusion: Acute pain episodes are the leading cause of ED visits and hospitalizations for those with SCD. Thus, to properly meet the needs of patients with SCD and improve their outcomes, there needs to be collaborative, standardized and SCD- specific clinical guidelines for ED providers to utilize. Pre-implementation of a standardized acute pain management algorithm, our institution was not meeting national guidelines for administration of analgesia and assessment of pain. Post-implementation results are currently under analysis.

Impact and Implications: Patients with SCD experience longer wait times in the ED and significant bias and mistrust regarding pain concerns. Standardized clinical guidelines have the potential to improve care and decrease variation in care delivery. The results from our study can inform institutional and national policy makers on gaps that exist in pediatric SCD, so that research funding can be appropriately allocated. 